\title{
LE MODÈLE POLITIQUE VÉNITIEN NOTES SUR LA CONSTITUTION D'UN MYTHE
}

\author{
Jean-Louis FourNEL
}

\begin{abstract}
RÉsumÉ : À partir du xvl ${ }^{\mathrm{e}}$ siècle, le gouvernement «mixte » de Venise devient, pour l'Europe de l'Ancien Régime, un modèle politique. Composant harmonieusement les principes de la démocratie (avec le Grand Conseil), de l'aristocratie (avec le Sénat) et de la monarchie (avec le Doge), garantissant la paix sociale et la stabilité des institutions, prétendant préserver Venise des aléas de l'Histoire, cette forme de gouvernement donne naissance à un véritable mythe. Volet essentiel de la réflexion sur la diversité des traditions républicaines italiennes, l'étude du mythe politique vénitien permet aussi de s'interroger sur les emplois discutables du paradigme républicain dans l'historiographie récente.
\end{abstract}

Mots-CLÉs : mythe politique, républicanisme, Venise, histoire des institutions, Renaissance.

ABSTRACT: From the sixteenth century on, the " mixed" government of Venice became a political model for the Europe of the Ancien Régime. Harmoniously combining the principles of democracy (the Grand Council), aristocracy (the Senate) and monarchy (the Doge), guaranteeing social peace and the stability of the institutions, claiming to protect Venice from the hazards of history, this form of government gave birth to a veritable myth. An essential part of the reflection on the diversity of Italian republican traditions, the study of the Venitian political myth allows us to question also the disputable usages of the republican paradigm in recent historiography.

KEYwORDS : political myth, republicanism, Venice, history of institutions, Renaissance.

Revue de synthèse : $4^{e}$ S. $\mathrm{N}^{\circ \mathrm{s}} 2-3$, avril-septembre 1997, p. 207-219. 
ZuSAMMENFASSUNG : Seit dem 16. Jahrhundert war die "gemischte Regierung" von Venedig ein politisches Modell für das Europa des Ancien Régime. Diese Regierungsform, bei der die Grundsätze der Demokratie (Großer Rat), der Aristokratie (Senat) und der Monarchie (Doge) harmonisch miteinander verbunden waren, garantierte den sozialen Frieden und die Stabilität der Institutionen. Sie erhob den Anspruch, Venedig vor den Wechselfällen der Geschichte zu bewahren und führte zur Entstehung eines wahren Mythos. Die in dieser Untersuchung angestellten Überlegungen über die verschiedenen Aspekte der republikanischen Traditionen Italiens eröffnen die Möglichkeit, die nicht unumstrittene Verwendung des republikanischen Paradigmas in der neueren Geschichtsschreibung kritisch zu hinterfragen.

STICHWÖRTER : politischer Mythos, Republikanismus, Venedig, Geschichte der Einrichtungen, Renaissance.

Jean-Louis FourNeL, né en 1959, enseigne l'italien à l'université Paris VIII. Il étudie l'histoire de la rhétorique dans l'Italie de la Renaissance et la pensée politique républicaine à Florence et à Venise au $x v^{c}$ et au $x v I^{c}$ siècle. Il a notamment publié, en collaboration avec JeanClaude Zancarini, plusieurs ouvrages de Savonarole et de Francesco Guicciardini.

Adresse : Université Paris VIII Vincennes-Saint-Denis, Département d'études italiennes, 2 rue de la Liberté, F-93526 Saint-Denis Cedex. 
Tout au long de ce que l'on a coutume d'appeler l'Ancien Régime', il est bien peu de voyageurs, d'ambassadeurs ou d'observateurs sociaux qui n'aient pas tiré de leurs séjours à Venise ou de leurs lectures sur l'histoire de la Sérénissime, analyses complexes ou remarques fragmentées sur la spécificité du régime politique en vigueur dans la république. Tout se passait comme si ce régime politique était perçu comme aussi exceptionnel que le site unique de la cité des lagunes, avec son rôle de pont et de ville frontière entre l'Orient et l'Occident. Venise était le seul état à récupérer à la fois l'héritage politique des Romains et celui des Grecs, et surtout à intégrer dans sa tradition l'héritage de tous les Romains et celui de tous les Grecs, au-delà des partitions traditionnelles entre les moments républicains et impériaux des deux référents historiques : en effet, elle pouvait se réclamer de la double tradition latine et byzantine, ces deux Romes successives auxquelles Venise tout ensemble empruntait et se mesurait sans pour autant jamais se poser simplement comme leur héritière, sans jamais rechercher un quelconque statut de «troisième Rome». L'Adriatique s'appela longtemps tout simplement le « golfe de Venise » et les Vénitiens purent même longtemps penser, avant la montée de la puissance turque, que « leur » mer, la Méditerranée, était paradoxalement le vrai centre de gravité d'un empire qui s'égrenait en comptoirs, en chapelets d'îles et en bandes côtières, empire sans frontières définies et dont l'étendue se mesurait à l'aune du rayon d'action des galères plutôt qu'à celle de la superficie d'un territoire circonscrit par un limes. La république de Venise, dont la puissance et l'influence naquirent d'abord du commerce et des relations avec l'Orient puis de l'image mythifiée qu'elle renvoyait d'elle-même, s'est ainsi toujours appuyée pour une bonne part sur des fondements immatériels, sur cette idée, cette image qu'elle parvenait à donner d'elle-même à l'intérieur et à l'extérieur. C'est pour cette raison qu'aborder la question du mythe de Venise ne signifie pas faire œuvre « littéraire ", " sémiologique » en quelque sorte, d'analyse d'une construction idéologique : le modèle en question a produit constamment des effets, il n'est pas un simple habillage de pratiques ou un leurre et il n'a jamais fonctionné comme une simple célébration d'un passé révolu ou d'une fondation légendaire. Il serait donc à cet égard trompeur et inadéquat de circonscrire l'examen à la trop classique opposition mythe/réalité pour traquer ce qui, dans les traces de l'his-

1. Et même au-delà si l'on songe à l'intérêt porté à la tradition politique vénitienne chez les intellectuels qui développèrent au XIx ${ }^{e}$ siècle une critique du libéralisme au nom de la théorie des élites. Voir, à ce propos, BURKE, 1988, p. 15-25. (Pour plus de précisions sur les références citées en notes, se reporter à la Bibliographie finale, p. 218-219.) 
toire, dément le modèle théorique diffusé. Ce qu'il faut en revanche tenter de faire, c'est rassembler des éléments pour une étude de la construction, des fondements, des principes et des effets du modèle mythique, s'interroger pour savoir ce qui le rend nécessaire à un moment historique donné, saisir son articulation avec d'autres modèles disponibles (et concurrents!) dans les mêmes champs, comprendre les formes que peut prendre sa circulation et, enfin, mettre en évidence les mutations que les migrations spatiales et temporelles font subir au mythe de Venise ${ }^{2}$.

Les enjeux de cette affaire par rapport à la question du républicanisme se situent sur un triple plan : sur le plan historiographique d'une contribution à l'enquête sur les nœuds essentiels d'une reconstruction de l'histoire du républicanisme, sur le plan méthodologique d'une distinction, encore plus nécessaire que dans d'autres cas, entre ce qui relève de l'histoire des idées ou des représentations et ce qui se rattache à l'histoire tout court ou à la philosophie politique et, enfin, sur le plan strictement critique et politique de la compréhension des implications idéologiques de certains travaux consacrés à cette question. En effet, la dernière victoire du mythe de Venise et la dernière démonstration de sa force, ne sont-elles pas manifestes dans la place que lui accorde une certaine tradition critique d'origine anglo-saxonne? Bref, ne faudrait-il pas tenter, pour échapper aux « lentilles déformantes " du mythe de Venise dénoncées par Robert Finlay ${ }^{3}$, de comprendre ce que fut ce mythe sans tout croire des discours d'autocélébration mais sans négliger pour autant ce qu'ils traduisent et ce qu'ils produisent?

Il existe des traces remontant au haut Moyen Âge de la défense et illustration du caractère unique et inimitable de la situation et de la tradition de Venise. C'est une des raisons pour lesquelles, lorsque l'on parle du " modèle vénitien " ou du « mythe de Venise ", certains historiens comme Gina Fasoli tendent à renvoyer à une chronologie toujours plus reculée dans le temps, en allant jusqu'à évoquer comme acte fondateur la récupération par des marchands vénitiens du corps de saint Marc en 827-8284. Il ne s'agit pas ici de rentrer dans un tel débat : s'il existe indiscutablement des éléments épars de constitution du mythe bien avant la fin du $x^{e}$ siècle, c'est à cette époque-là qu'on peut vraiment commencer à parler de l'édification d'une image globale très cohérente de l'ordre républicain vénitien

2. Il va de soi que je me limiterai ici, sans pouvoir prétendre faire davantage, à une mise en place des frontières et des enjeux d'un programme de travail qui a déjà donné lieu à plusieurs ouvrages et à maintes controverses : voir la section Études de la Bibliographie, infra, p. 218219. Pour une mise au point bibliographique récente très utile sur le « mythe de Venise », voir Sil.vano, 1993, en part. p. 9-38.

3. FINLAY, 1982, p. 17.

4. FASOLI, 1974. 
tendant à se présenter comme un modèle imitable, cet «ensemble de notions relatives à la perception de soi et du propre prestige de la République » qui fait le mythe de Venise selon Robert Finlay ${ }^{5}$. Cette phase de constitution du mythe culminera cinquante ans plus tard avec la publication en latin, suivie très rapidement de traductions dans les principales langues vulgaires du temps (français, anglais, italien), du traité de Gasparo Contarini, De magistratibus et republica venetorum ${ }^{6}$. L'influence de ce traité fut considérable et il est vrai, comme l'affirme Gigliola Fragnito ${ }^{7}$, que l'ouvrage assura à l'auteur une renommée que son chapeau de cardinal et ses maints écrits théologiques n'auraient jamais pu lui procurer. Il sera suivi pendant deux siècles par une série d'ouvrages qui contribuent à diffuser une image idéale de la république : du dialogue du Florentin Donato Giannotti, Della repubblica de' viniziani - écrit entre en 1525 et 1527 mais publié en 1540 - aux ouvrages de Francesco Sansovino ou Paolo Paruta, des volumes de Sebastiano Erizzo à ceux de Casanova ou Romanin - ces deux derniers étant hérissés par les critiques des Français qui, à partir du milieu du XVII siècle - d'Amelot de La Houssaye à Daru condamnent le despotisme vénitien.

Toutefois, à partir de la fin du $\mathrm{XvI}^{\mathrm{e}}$ siècle, le mythe de Venise est essentiellement répétitif et alimenté par l'émergence d'une sorte de «contremythe », à partir des critiques de Jean Bodin puis surtout de la violente campagne anti-vénitienne déclenchée au moment de l'interdit de Paul V contre Venise en $1605^{\circ}$. Nous nous en tiendrons donc à quelques remarques sur le mythe de Venise au $x v^{\mathrm{e}}$ siècle.

Durant la première moitié du $x v^{e}$ siècle, Venise, tirant le meilleur parti de l'«alliance des républiques » nouée avec Florence pour faire face au danger de la «tyrannie » incarnée par les ambitieux ducs de Milan de la famille Visconti, avait mené à bien en quelques décennies la conquête de territoires toujours plus étendus sur la terre ferme. Ce n'est, en effet, qu'au Quattrocento que Venise entreprit vraiment de bâtir son «état de terre ferme » en partant à la conquête de Padoue, Vicence, Vérone, Bergame ou Crémone. Parallèlement, malgré quelques défaites navales face aux Turcs, Venise étendit sa domination vers l'est jusqu'à l'île de Chypre, annexée en 1489. De ce fait, dans les institutions de la Sérénissime, s'inscrivit en même temps une partition entre lo stato de mar et lo stato de terra, lourde d'un débat susceptible de diviser la cité entre ceux qui privilégiaient la

\footnotetext{
5. FinLAY, 1982, p. 17.

6. Contarini, 1543 .

7. Fragnito, 1988.

8. Voir [ANONYME], 1612, Squittinio della libertà veneta : un des textes les plus importants de cette campagne anti-vénitienne, qui se développe beaucoup en France de Bodin à Rousseau en passant par Amelot de La Houssaye ou Saint-Didier.
} 
défense de l'empire d'outre-mer et ceux qui voyaient l'avenir de la république se jouer sur la terre ferme.

Malgré tout, à cette époque-là, se développe une image de Venise comme le modèle d'une stabilité et d'une paix sociale que bien peu d'États italiens connaissent en ces temps de conjuration, de coups d'État et de querelles dynastiques. La longévité des institutions sans changement notable, la conservation immuable des formes extérieures du pouvoir, la concorde qui semblait régner dans la cité étaient au centre de ces éloges de la république de Venise qui semblait atteindre cet objectif d'unité harmonieuse et de cohésion politique typique de la pensée aristotélico-thomiste.

C'est ce qui a fait dire à Jacob Burckhardt que Venise était « la cité du silence politique ", par opposition à une Florence tourmentée et agitée sans cesse par les luttes sociales ${ }^{9}$. Cette formule est aujourd'hui vertement dénoncée par une critique américaine soucieuse - au nom de la défense et illustration d'un républicanisme italien ancêtre des sociétés politiques anglo-saxonnes - de gommer les différences entre les deux grandes républiques ${ }^{10}$. Cependant, quelque schématique que puisse être l'opposition faite par J. Burckhardt, on peut du moins souligner qu'elle était ressentie exactement de cette façon par nombre de témoins du moment, parmi lesquels certains étaient aussi peu démunis de capacités d'analyse qu'un Machiavel ou un Guichardin... Le parallèle entre les deux républiques était fréquent et donnait même lieu à une sorte de typologie comparée ${ }^{11}$. Les admirateurs de la Sérénissime se plaisaient à noter ainsi que, si les deux républiques pouvaient se réclamer de la libertas, d'un populus - dont l'unité est fondée sur l'égalité entre citoyens - et des vertus républicaines, seule Venise possédait l'imperium, la nobilitas, la tempérance et la capacité paradoxale à conquérir dans la paix, en étant acceptée par ses nouveaux sujets. Ce n'est qu'à Venise que put d'ailleurs se faire jour une sorte de religion de l'État dès le XIv' siècle : l'État n'appartenait pas à une famille ou à un groupe de familles et il dominait l'individu qui ne devait avoir d'autre ambition que de servir la république en acceptant toute fonction que celle-ci décidait de lui confier.

Dans ce cadre-là, ce ne sont pas les vertus du citoyen et leur éloge protoou préhumaniste et humaniste qui contribuent à renforcer la république mais l'existence de la république qui permet au citoyen de se montrer vertueux, voire qui le contraint à le faire. Venise peut ainsi se poser, nouveauté étonnante à l'automne du Quattrocento, en rivale potentielle de la Rome antique, dans une sorte de première querelle politique des Anciens et des Modernes qui, si elle n'apparaît très explicitement qu'avec

9. BURCKHARDT, 1986, t. 1, p. 96.

10. C'est le cas, explicitement, de Bouwsma, 1968 mais aussi de Pocock, 1975.

11. Voir TATEO, 1990, p. 181 sqq. 
Contarini et surtout Paruta, est d'emblée selon nous l'impensé et le non-dit du développement du mythe de Venise. D'ailleurs, contrairement à Florence et à la plupart des républiques citadines italiennes, le mythe fondateur de Venise ne cherche pas de légitimité particulière chez de glorieux ancêtres (Romains, Lombards ou Francs) mais se définit historiquement contre tout ancêtre particulier: les Vénitiens auraient fondé leur cité au $\mathrm{vI}^{\mathrm{e}}$ siècle dans les îles de la lagune parce qu'ils refusaient l'assujettissement aux envahisseurs barbares. De même, cas unique en Europe, ils échappent à tout système médiéval d'investiture et ils ont très vite pris leurs distances par rapport à leur seul tuteur - l'empire byzantin (tuteur vite effacé des mémoires citadines, bientôt leur protégé et dont ils feront piller la capitale lors de la $4^{\mathrm{e}}$ croisade). De ce fait, la liberté originelle de Venise devient la première pierre sur laquelle construire un mythe républicain spécifique, dans l'affirmation d'une différence radicale, d'une unicité et d'une identité incomparables.

Florence, Rome et Venise, c'est entre ces trois modèles, entre ces trois termes que se constituent les enjeux du débat sur le régime vénitien. C'est ce que comprennent parfaitement les Florentins qui intègrent la référence vénitienne, plus ou moins modifiée et manipulée, à la plupart des débats sur la réforme de leur gouvernement, d'abord pour soutenir, comme l'a montré Felix Gilbert ${ }^{12}$, une solution aristocratique puis, avec Savonarole, pour fonder une alternative plus populaire ${ }^{13}$. C'est aussi ce qui explique que la critique du régime vénitien s'avère une constante de la pensée machiavélienne : il est difficile de louer en même temps Rome et Venise car les deux modèles s'opposent terme à terme comme la guerre s'oppose à la paix, comme une république tirant sa vitalité des conflits et de la conquête s'oppose à une autre république qui théorise l'effacement de toute conflictualité sociale et la vertu première du maintien de la paix, comme les citoyens soldats s'opposent à des marchands commerçants qui s'appuient sur des troupes mercenaires et des généraux qui, pour leur armée de terre, ne sont jamais des Vénitiens, comme, enfin, s'oppose la vertu dynamique des Romains à la prudence habile des habitants de Venise qui tirent parti de la fortune plus qu'ils ne savent la bousculer. Bref, il est légitime de se poser la question de savoir si le renforcement et la reprise du mythe de Venise ne peut pas être analysé par moments comme une des formes que va prendre l'antimachiavélisme.

Il n'est pas étonnant de voir dès lors que les réactions respectives de Florence et de Venise, face à la crise politico-militaire qui frappe de plein fouet la péninsule à partir du début des guerres d'Italie, en septembre 1494,

12. GILBERT, 1968.

13. Voir, à ce propos, Savonarole, 1993. 
ne sont pas du même ordre. À Florence, on recherche une articulation inédite entre l'état d'urgence (lo straordinario) et la réforme sans se contenter de revenir purement et simplement aux formes ancestrales de l'antico vivre popolare du comune alors qu'à Venise prévaut l'approfondissement des éléments constitutifs du mythe dans une direction plus institutionnelle. Avant cela, Venise en effet s'était vu infliger une déroute d'une gravité sans précédent qui avait fait perdre à la république - après la bataille d'Agnadel le 14 mai 1509 - la quasi-totalité de ses possessions de terre ferme. La réaction vénitienne fut de deux ordres, militaire - dans un premier temps - puis idéologique et politique. En effet, moins de deux mois après Agnadel, grâce à l'appui du petit peuple de terre ferme et au sursaut patriotique des jeunes nobles de la Dominante, Venise reconquiert Padoue à la surprise de tous les contemporains puis, en quelques années, récupère toutes ses possessions jusqu'aux confins de la Lombardie. Parallèlement, la république de Venise promeut une image d'elle-même dans laquelle la reprise de certains thèmes déjà anciens va de pair avec une réelle inflexion du discours politique : la défense et illustration du régime vénitien passe désormais par la présentation de Venise comme le modèle même du gouvernement tempéré et «mixte ${ }^{14}$.

Selon ce discours nouveau, la Sérénissime combinait harmonieusement les principes monarchique, aristocratique et démocratique avec respectivement le Doge, le Sénat et le Grand Conseil. Dans leur infinie sagesse, les aieux des Vénitiens avaient fait, d'un côté, de l'attribution d'un rôle déterminé à tous les habitants et, de l'autre, de l'égalité entre les nobles, ou «patriciens», les fondements de la république. Les institutions étaient d'ailleurs particulièrement sourcilleuses sur le respect de la cohésion sociale qui conférait à chaque groupe social sa place et sa fonction dans la vie de la cité, instaurant, par exemple, avant tout autre pays d'Europe, une politique d'assistance systématique, gérée par l'État, pour les citoyens les plus pauvres. Tous les membres des familles nobles dont les noms figurent dans le livre d'or, où les naissances et les mariages de nobles sont consignés, ne portent pas de titre particulier sinon celui de gentilhomme (Nobilhuomo, N.H.). Ils ont tous à partir de l'âge de vingt-cinq ans une place à vie au sein du Grand Conseil, sauf s'ils choisissent d'entrer dans les ordres ou de se faire prêtre, et il en ira de même pour leurs enfants, à la seule condition qu'ils ne dérogent pas en se mariant avec une « roturière ". Tous représentent une voix et une seule lors des élections qui ont lieu dans le Grand Conseil et tous sont éligibles : chacun a le droit de prétendre à être élu pour telle ou telle fonction publique et personne ne peut se soustraire à

14. Gasparo Contarini manifeste parfaitement l'importance qu'a pu avoir le traumatisme d'Agnadel pour sa réflexion, en faisant de cet événement la seule référence à l'histoire contemporaine contenue dans son traité. Voir aussi Cervelu, 1974. 
son devoir quand il est choisi par ses pairs, sous peine de lourdes amendes, voire de l'exil ou de la prison.

Tout au long des cinq livres du gouvernement, Contarini nous explique ainsi complaisamment par le menu le complexe système de vote dans chacune des instances du système de gouvernement ou expose le rôle et la composition de la moindre des magistratures nommées par ces conseils. La politique semble ainsi se ramener à un pur problème de droit public aux dépens de tout examen des pratiques de gouvernement et de la question sociale : la guerre et le conflit sont bannis de l'exposé dans une image récurrente d'harmonie musicale qui ajoute une touche platonicienne au système aristotélicien. Pour Contarini, les républiques antiques de Sparte ou de Rome ont justement eu le tort d'être tournées sans cesse vers la guerre. Il va même jusqu'à supposer une incompatibilité entre le maintien de la paix civile et l'importance accordée à l'organisation militaire dans les institutions républicaines car les citoyens soldats fomentent volontiers des séditions lorsqu'ils n'ont plus à défendre les frontières de la république : tout se passe comme si, pour Contarini, les combats extérieurs menés directement par les citoyens préparaient les futures guerres civiles.

$\mathrm{Au}$ contraire, à Venise, la justice et la loi règnent dans la paix et la concorde : le régime vénitien devient ainsi naturellement parfait dans une construction très liée à l'esprit de la Renaissance finissante, où l'homme atteint la perfection lorsqu'il imite le mieux la nature et la création divine. Pour Contarini, les lois vénitiennes permettent même le contrôle des passions humaines. En effet, la loi produit de la vertu (alors que, chez Machiavel, c'est au contraire la vertu qui permet l'instauration des bonnes lois) et fonde l'égalité (qui n'est pas d'ailleurs la participation de tous au gouvernement mais seulement l'égalité devant la loi). Dans ce cadre-là, la corruption (concept central chez Machiavel pour expliquer la dégénérescence des régimes et la nécessité dans certains cas du retour à des moyens extraordinaires) n'est plus une catégorie pertinente et productive, puisque la principale qualité de l'ordre vénitien est de résister au temps. Mais ces institutions naturelles ne sont pas une pure application des propositions théoriques des Anciens car les Vénitiens, grâce à leur sagesse pragmatique, n'ont fait les choses qu'à l'image des modèles possibles : la ressemblance avouée n'affiche ainsi qu'une proximité qui ne se veut pas identité et le régime qui appartient au monde contemporain garde son autonomie et sa spécificité par rapport aux glorieuses références des mondes du passé. À cet égard, au-delà de l'accent mis sur les particularités du site de la république, ce sont les développements consacrés au « conseil des Dix » ou aux Avogadori (magistrats importants servant d'accusateurs publics) qui introduisent le mieux le décalage entre la théorie du gouvernement exposée par Contarini et le modèle classique du gouvernement «mixte». Les bons 
docteurs vénitiens ont inventé les Dix pour en faire le remède aux possibles maladies du corps social, un baume permettant de soigner les « humeurs » différenciées selon les groupes de citoyens - ces mêmes « humeurs » qui, préludant à l'expression violente des passions et des désirs des citoyens, sont un des éléments essentiels de la dynamique de l'histoire machiavélienne. De même, les Avogadori ne sont pas, selon Contarini, comparables aux tribuns de la plèbe chers à Machiavel. Venise a, tout compte fait, un gouvernement meilleur que celui des Romains parce que, aux façons des Anciens, les Vénitiens ont ajouté la tempérance. Le peuple peut accepter de ne pas prendre part à la gestion des affaires car la république lui garantit un bon approvisionnement et une justice équitable. La meilleure preuve de la supériorité de Venise sur les républiques modernes et anciennes, c'est le fait que, d'une part, depuis mille deux cents ans la Sérénissime n'a jamais été dominée par une puissance étrangère et que, d'autre part, elle n'a pas souffert - contrairement à la majorité des comuni de la péninsule italienne - les maux des « discordes civiles » et des « tumultes » populaires.

Mais, paradoxalement, le premier ouvrage publié consacré au gouvernement ne fut pas le traité de Contarini mais un dialogue inachevé écrit par un « étranger », le Florentin républicain Donato Giannotti, qui fit éditer dès 1540 son Della repubblica dei Viniziani, écrit entre 1525 et 1527 alors que l'auteur est en semi-exil à Padoue et à Venise. Le travail de Giannotti est le pendant indispensable au traité de Contarini. Moins « idéologique » et plus libre de son propos que le noble Vénitien, Giannotti historicise le discours du mythe et met en évidence les problèmes de fonctionnement du système institutionnel vénitien en décrivant les brogli, la corruption et les dissensions internes au patriciat. En effet, il applique à la pensée politique et à l'histoire du droit public vénitien les principes méthodologiques de l'enquête philologique que son éducation humaniste lui a inculqués, pour reconstituer pièce par pièce le puzzle de l'histoire du « gouvernement des Vénitiens ». Les institutions n'ont aucune caractéristique «naturelle» et n'existent qu'en action : chaque instance doit être étudiée dans son fonctionnement pratique et suivant la ligne des rapports croisés et incessants qu'elle entretient avec les autres instances du régime, sans toutefois les examiner à la lumière d'une histoire contemporaine étrangement absente dans l'ouvrage de Giannotti. Un tel discours technique sur les institutions n'a pas d'avenir à court terme car ni les théoriciens de la souveraineté à la Bodin ni ceux de la raison d'État à la Botero n'en ont besoin. Giannotti interrompt l'écriture de ce dialogue en 1527 pour rentrer à Florence mettre ses compétences au service du dernier gouvernement républicain florentin. Disciple de Machiavel, dont on sait les réticences à l'égard du régime vénitien, et, malgré tout, admirateur de Venise, Giannotti n'inscrit pas son discours dans un propos édifiant. En effet, son approche «administrative» 
du gouvernement - lucide et historique —, au lieu d'alimenter le mythe, inaugure une autre voie pour les textes sur le régime vénitien car la Sérénissime y apparaît moins comme un modèle à suivre ou à reproduire que comme l'étonnant produit, unique, contradictoire et complexe, d'une conjonction de facteurs aussi fragile qu'exceptionnelle. Sans que cela fasse le moins du monde partie du projet de Giannotti, une telle posture permet d'asseoir les fondements d'un contre-mythe qui va se développer principalement au XVII ${ }^{e}$ siècle et surtout en France. Il opposera au discours vénitien un discours quasiment symétrique, où les énoncés se répondent terme à terme, la liberté originelle se traduisant en servitude démontrée, la cohésion postulée en dissensions réelles, l'honnêteté et les valeurs civiques en corruption et en intérêts familiaux bien compris, la virtù en fortuna, le règne de la loi en tyrannie despotique du sombre conseil des Dix... Le " contremythe » ne peut dès lors être que l'ultime victoire du mythe puisqu'il reste sur le terrain choisi par ce dernier.

Pendant ce temps, Venise use ses dernières forces à résister jusqu'au bout à l'avancée ottomane en Méditerranée, île par île, sans pouvoir jouer alors un rôle significatif sur l'échiquier européen, où dominent désormais les grandes monarchies nationales en lutte au rythme des aléas de la rivalité entre les maisons de France, d'Espagne et d'Autriche. La diffusion du mythe du régime politique vénitien dans toute l'Europe et l'enfermement de la république dans une prudente politique de neutralité sont les deux faces de la même médaille. La proposition politique, qui s'était voulue actuelle et contemporaine au $\mathrm{XvI}^{\mathrm{e}}$ siècle, va se perdre dans les chemins de traverse menant soit à des refuges utopiques ou décadents ${ }^{15}$ soit à des utilisations du paradigme républicain dans le but d'effacer un peu trop aisément conflits et contradictions de l'histoire républicaine ${ }^{16}$.

Jean-Louis FouRNEL

(novembre 1995).

15. Sur ce point, voir Fontana, 1989, p. 146-181.

16. Développer sérieusement ce dernier point, ouvertement polémique à l'égard de certains glissements d'une tradition critique anglo-saxonne d'études sur le " republicanism » florentin ou vénitien (je pense à des auteurs comme Bouwsma, Pocock, Lane ou Skinner), m'entrânerait vers un trop long exposé et je renverrai donc, pour ce faire, à d'autres moments et à d'autres lieux... Voir, sur cette controverse, PECCHIOL, 1983. 
BIBLIOGRAPHIE

\section{Sources}

Amelot DE LA Houssaye (Abraham-Nicolas), 1676, Histoire du gouvernement de Venise, Paris.

Amelot de LA Houssaye (A.-N.), 1677, Supplément à l'Histoire du gouvernement de Venise, Paris.

[Anonyme], 1612, Squittinio della libertà veneta, La Mirandole (trad. franç. par A.-N. AMELOT DE LA HousSAYE sous le titre Examen de la liberté originaire de Venise, Paris, 1677).

Burke (Peter), 1988, $1^{\mathrm{T}}$ éd., Venice and Amsterdam. A study of seventeenth-century elites, 1974, ici trad. ital., Ancône, Transeuropa.

Casanova (Giacomo), 1769, Confutazione della storia del governo veneto d'Amelot de La Houssaye, Amsterdam.

Contarint (Gasparo), 1543, De Magistratibus et Republica Venetorum libri quinque, Paris, ex officina Michaelis Vascosani (trad. ital. et franç. publiées à Paris en 1544).

Daru (Pierre A. N. B.), 1819, Histoire de la république de Venise, Paris.

Giannotn (Donato), 1540, Della republica de' Viniziani, Rome, Blado, éd. modeme par Furio Diaz, in D. GiannotT, Opere politiche, Milan, Marzorati, 1974, p. 27-152.

LiMOJON DE SAINT-DidIER (Alexandre-Toussaint), 1680, La Ville et la république de Venise, Paris.

PARUTA (Paolo), 1599, Discorsi politici nei quali si considerano diversi fatti illustri e memorabili di principi e di republiche antiche e moderne, Venise.

Romanin (Samuele), 1853-1861, Storia documentata di Venezia, Venise.

Sansovino (Francesco), 1556, Tutte le cose notabili e belle che sono in Venetia, Venise.

Sansovino (F.), 1580, Venetia città nobilissima et singolare, Venise.

Savonarole (Jérôme), 1993, Sermons, écrits politiques et pièces du procès, textes trad., annot. et prés. par Jean-Louis Fournel et Jean-Claude Zancarin, Paris, Seuil.

\section{Études}

Bertelli (S.), Rubinstein (N.), SMith (C. H.), éd., 1979, Florence and Venice. Comparisons and Relations, 2 vol., Florence, La Nuova Italia.

BESTA (Enrico), 1899, Il Senato veneziano : origine, costituzione, attribuzioni e riti, Venise.

Bock (Gisela), Skinner (Quentin), VIRoli (Maurizio), éd., 1990, Machiavellism and republicanism, Cambridge, Cambridge University Press. 
Bouwsma (William J.), 1968, Venice and the defense of republican liberty, Berkeley, University of California Press.

BurCKhaRdT (Jacob), 1986, Civilisation de la Renaissance en Italie, Paris, Le Livre de poche.

Cervelli (Innocenzo), 1974, Machiavelli e la crisi dello stato veneziano, Naples, Guido.

CEssi (Roberto), 1981, Storia della repubblica di Venezia, Florence, Giunti Martello.

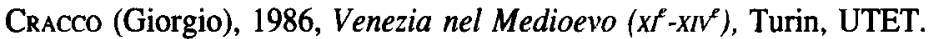

FAsoli (Gina), 1974, "Nascita di un mito ", in Scritti di storia medievale, Bologne.

Finlay (Robert), 1982, $1^{\text {re }}$ éd. Politics in Renaissance Venice, New Brunswick, Rutgers University Press, 1980, ici trad. ital., Milan, Jaca Book.

FonTANA (Alessandro), 1989, "La verità delle maschere » et « La città ritrovata ", in Il vizio occulto, Ancône, Transeuropa.

Fontana (Alessandro), Saro (Georges), éd., 1997, Venise 1297-1757. La République des castors, Paris, ENS Éditions.

FRAGNITO (Gigliola), 1988, Gasparo Contarini. Un magistrato veneziano al servizio della cristianità, Florence, Olschki.

GILBERT (Felix), 1968, « The Venetian constitution in Florentine political thought », in Florentine Studies, éd. Niccolai RuBinstein, Londres, Faber and Faber.

Hale (J. R.), éd., 1973, Renaissance Venice, Londres.

Lane (Frederic), 1973, Venice. A maritim Republic, Baltimore, John Hopkins University Press (trad. franç., Paris, Flammarion, 1985).

Maranini (Giuseppe), 1927 et 1931, La Costituzione di Venezia, 2 vol., Venise (rééd., Florence, La Nuova Italia, 1974).

Pecchioli (Renzo), 1983, Dal mito di Venezia all'ideologia americana, Venise, Marsilio.

Pocock (John Greville Agard), 1975, The Machiavellian Moment. Florentine political thought and the Atlantic tradition, Princeton, Princeton University Press (trad. ital., Bologne, Il Mulino, 1980, et trad. franç., Paris, Presses universitaires de France, 1997).

Pullan (Brian), 1971, Rich and poor in Renaissance Venice, Oxford, Blackwell (trad. ital., Rome, Il Veltro, 1982).

Queller (Donad E.), 1986, The Venetian patriciate. Reality versus Myth, Champaign, IL, University of Illinois Press (trad. ital., Rome, Il Veltro, 1987).

Silvano (Giovanni), 1993, La "repubblica de' Viniziani". Ricerche sul repubblicanessimo veneziano in età moderna, Florence, Olschki.

TATEO (Francesco), 1990, I miti della storiografia umanistica, Rome, Bulzoni.

ZoRDAN (Giorgio), 1980, L'Ordinamento giuridico veneziano, Padoue, CLUP.

En outre, on se reportera utilement aux différents volumes de la momumentale Storia della cultura veneta, coord. par Manlio Pastore STосCH et Girolamo ARNALDI, Vicence, Pozza, 1976, ainsi qu'à la Storia di Venezia, en une vingtaine de volumes de l'Istituto dell'Enciclopedia Italiana de Rome (les deux premiers volumes - l'un chronologique sur le $\mathrm{XVI}^{\mathrm{e}}$ siècle et l'autre thématique sur «Il Mare » sont parus en 1995). 